\title{
Philosophic aspects of developing new knowledge under data intellectual analysis (Big Data)
}

\author{
Bodrov A.A., Ramzaev V.M.
}

International Market Institute Samara

\begin{abstract}
Based on interdisciplinary analysis, the paper considers the process of forming new philosophy of knowledge, which is currently emerging owing to the use of information technologies and, above all, the Big Data techniques. Examined are also transformations of ontological and gnoseological paradigms of the scientific cognition contemporary system. Offered are new conceptions enabling comprehensive interpretation of diversity of natural and social factors influencing the human being as the processes owner, use of unobvious correlations in philosophic and social-economic practice. Access to these conceptions actualizes appropriateness of analysis with the aid of Big Data in the most significant spheres of the people's biosocial activity.
\end{abstract}

Keywords: Big Data technologies, spheres of bio-sociological human activities, ontological reality development, epistemological potential, virtual setting.

Citation: Bodrov A.A., Ramzaev V.M. Philosophic aspects of developing new knowledge under data intellectual analysis (Big Data). Proceedings of Information Technology and Nanotechnology (ITNT-2015), CEUR Workshop Proceedings, 2015; 1490: 338-345. DOI: 10.18287/1613-0073-2015-1490-338345

Ontological manifestations of reality in the XXI-st century are notable for extreme diversity, which often complicates its perception and disorients the people misleading them as to trueness of objective reality manifestation.

Under these conditions, virtualization becomes inherent not only to the social-andeconomic reality (which has been covered by the researchers quite a lot), but also the problem-bearing science field embracing ontological and gnoseological components, undergoing major transformations requiring philosophic and general-scientific comprehension.

In our opinion, the Big Data techniques can provide assistance to the human beings; these techniques make it possible to process and use huge information volumes, promote taking managerial decisions in all life spheres, change the world perception due to reality digital analysis as well as its ontological fundamentals, thus actualizing the necessity of revision of philosophic and general-scientific reality principles; it is equally true with regard to seemingly established 'material-spiritual' 
dilemma. Indeed, being part of the contemporary social-cultural system, Big Data actively impact all the present-day society's spheres [May14], transforming them in the human state of awareness, which enables reconsideration of the classical philosophic dilemma 'substance-spirit' offering instead a much more topical one: 'material-informational'.

Such dynamically changing conditions and a huge information flow strongly force the human beings to mediate upon the pattern of behavior in the information space, handling this space and comprehending the development in general; primarily comprehension is critical with regard to social development and its representation: either by real transformation of the substantive being or by interactive manipulations embodied only in the virtual medium (which actually stands for digital medium) being of high relevance to the human beings.

Speaking about the philosophic aspects of practical implementation of the Big Data techniques potential, it is immediately advisable to single out two main aspects of their possible application - ontological and gnoseological, mentioning in this connection that Big Data represent not just another manifestation of the information space, but a new philosophy enabling reception of knowledge on the basis of largescale data flow analysis.

In the ontological aspect, willingness to substitute real processes by virtual interactions becomes quite evident. Thus and so, excessive information does not always play a positive role, because in practice it can facilitate taking erroneous decisions, while in the philosophic sense it leads to emerging of virtual worlds (potential possibility of detalization of which is represented by Big Data), which ultimately facilitates deviation of the people from substantial reality. However, by changing perception of reality in its ontological manifestations the Big Data techniques enable large-scale visualization of social and natural processes in the interactive format, thus detailing the visualized objective reality while producing all sorts of models including economic ones. Undoubtedly, it leads to consolidation of perceptions of the objective reality's interactive nature.

In the context of the narrative it should be noted that the temporal presentation of classical science are subject to revision, but rather, a fundamentally new in-depth analysis on the basis of the potential detected by the use of Big Data technology.

It is worth noticing the increase in the variability of conceptual reality, multidimensionality of its capabilities, out of reach for philosophical and general scientific analysis without relying on the potential of Big Data.

In its ontological essence of Big Data technology actually form a fundamentally new coordinate system and the nature of modern society, which displaces an established understanding of the world. However, previous performance and characteristics of bio- and geo- systems do not disappear, but they are forced out by much more detailed new systems, capable of providing virtually sensory reflection of reality. The line between the virtual and sensual at the same time is disappearing; putting the person in a confusion asking a quite simple question: whether he has a true reality? One of its most important, as we can say "classic" characteristics is time, which has an ability that is worth noting separately, to accelerate and decelerate in a social sense, depending on the degree of saturation by socially significant events. 
Each event, which is considered through the prism of Big Data, can be become an important, vital, revolving. Therefore, it should be stated clearly that now we face unprecedented acceleration of social time.

Thanks to Big Data technologies the primacy of permanently accelerating time makes the search for the cause of human reality a luxury, revealing the danger that the reality is transformed into an impersonal "white" space.

Knowledge of individual industries are integrated into a single system that visibly indicates the formation of integral knowledge, integrated in all its branches and directions and is able to respond to requests quickly, comprehensively and accurately, leading the search for the causes outside of the Big Data technology. And if earlier the reality had quite a limited number of variations of its epistemological perspectives, now the Big Data let you create countless variations of the potential realities represented in the digitized space-time models.

The Big Data techniques gnoseological potential deserves special attention; it can embrace all the scientific investigations stages - from research problem statement up to extension of the research outcomes [Sad14].

Special emphasis shall be made on the possibility of introducing changes in the key cognition methods with the aid of Big Data, which would help overcome crisis in many humanities, thus producing absolutely new scientific theories under the use of enormous array of diverse information. Attempts to use this approach with regard to analyzing the world history are undertaken at the Moscow State University. Detalization of the history-related reality turns out to be so multidimensional and thorough that within the framework of the interactive history visualization 'ChronoZoom' project it is entirely possible to pool together information on social events of the past, climate, geographical changes and genealogical data of individuals [Ber14]. However, addressing mainly the historical process formal detalization the philosophy of history is almost completely ignored.

Spreading of the Big Data possibilities in the gnoseological sphere makes it quite realistic to reconsider and expand the coherence notion as one of the criteria of scientific truth, practical realization of which implies correlation of each new scientific statement with the previously made statements within the framework of the given theoretical system. Application of the Big Data potential in this direction is practically inexhaustible.

The Big Data universal nature makes it possible to interpret the respective techniques in a broad spectrum of their potential capacity, which is extremely topical for the gnoseological sphere. That sort of consideration enables presentation of the Big Data simultaneously as a tool of scientific hypotheses publication, elicitation of facts and regularities in combination with their novelty validation as well as a tool of disseminating the research results.

Analysis of the potential of Big Data, an updated process of implementation of these technologies in the industrial and scientific practice, convinced us visibly of the need to use the axiological approach to the perception of the likely impact of technology on society beginning of XXI century. The background, providing the possibility of problematization of the process of interest is fairly simple to identify. In our opinion, it is in the situation, where the inner content perfectly correlates with the 
biblical mythology that characterizes God as the omniscient beginning. It is the characteristics that will interest us, first of all, due to the fact that Big Data technology allows us to thoroughly detail the reality of man in elevating the status of being omniscient, thanks to the fundamental possibility of cloning able to create their own kind artificially.

This significant increase in the person's status really increases the potential impact on the environment, and the creatures of their own kind, setting at the same time before the man an extremely significant problem. They are defined by the opposite way; oriented meaningful vectors directed both from the surrounding reality, the perception of which is now more mediated by Big Data, and from the man as a creature possessing sufficient capacity to transform the world on your own.

Detailing these problem areas, we believe that it is important to note a number of highlights: gaining the ability to smallest, and probably the most precise detail of reality with the help of Big Data, including in terms of its potential development, will a man be able to "face the truth" if the forecast is extremely unfavorable, will he have the will and reason to overcome the uncertainty of the future and detailed Big Data problems, as all predictions based on Big Data, may affect his immediate personal interests, which a priori cannot be taken impartially? In the perspective it should also be determined who will have access to this kind of analysis and prediction, primarily of negative character, because one of the fundamental characteristics of scientific knowledge is accessibility to all, and it is closely associated with humanity, while the negative information is able to deprive the community from the balance, therefore, access to it should be limited.

Such-like reasoning touches not only philosophic spheres, but also the real economy sector, where balanced solutions are of great demand, where increase in goods and services sales volume is critical, i.e., what is meant here is managing the consumer behavior and primarily - forecasting for at least the short-term perspective the customers' preferences (as a special case).

Complexity of such forecasts is quite evident and seemingly formidable, which is largely determined by a huge diversity of all sorts of factors influencing the human beings as acting parties.

In terms of the sociocentrism principles, it is necessary to integrate the human being into the geosocial space from the standpoint of the XXI century science - in the status of collective subject with the purpose of establishing correlation between the individual and group conduct causality and primarily as to individual motivations (both deliberate and unconscious), which are often conditioned by geomagnetic processes of the surrounding media. That sort of task is extremely topical not only from the scientific point of view, but also in view of the complex optimization of all the social-economic system interrelations, specifically pertaining to their management. The abovementioned task can be successfully settled under application of large-scale diverse information field (of social and nature-specific kind) - by using the Big Data mechanisms.

This being so, it is quite possible to reveal and fully utilize (both in philosophic and social-and-economic practices) correlations that are not always evident. In such a way, the Big Data approaches - owing to analyzing huge information volumes - 
make it possible to establish dependence of the social-and-economic manifestations of the people's life activities on the macro- and mega-processes proceeding in the geoand helio-systems, thus demonstrating the possibility of perceiving the people's economic activity as a kind of derivative with respect to a number of factors, of which attention should be paid primarily to the non-cyclic factors emerging in the Sun; the 28-day cycle of the Moon's activity; annual, seasonal and daily solar cycle; the 11year cycle of Sun's activity linked to periodicity in the motions of the Sun's spots. The potential of such information - as indicators in the economic simulation approaches - is at present far from being properly studied, although the abovementioned phenomena have been long known in natural science. By way of giving an example, the 11-year cycle of the solar activity was discovered by G. Schwabe as early as in mid of the XIX-th century. Dwelling on the relations between the people and the space global entity, the Russian cosmist K.E. Tsiolkovsky wrote: 'Everything is generated by the Universe. It is the beginning of all things, everything depends on it ... The most intelligent creature fulfills only the will of the Universe. This will, depending on the senses cannot be the sole source of acts: the Universe can always intervene, distort, disrupt, and not allow fulfilling the will of one mind. We say all depends on us, but we ourselves are the creation of the Universe. Therefore, it is better think and say that it all depends on the Universe. We believe, but the Universe manages it as it wants, without ceremony it destroys our plans, and even destroy the entire planet with its intelligent beings. If we succeed and fulfill our will, it is only because the Universe allowed this to us. It always has at its disposal many ways and causes to retard our activity and manifest another, a higher will, although our will is just the will of the Universe'. [Tsi28] Close interrelation of the mankind and our planet's biogeosphere was pointed out by V.I. Vernadsky paying attention to the fact that 'the man and the mankind are first of all, and most closely, linked with the living matter populating our planet. By no real physical process can they be separated from it... The man as any other living natural (or real) body is inseparably linked to a certain geological envelope of our planet, with the biosphere, noticeably differing from its other envelopes. The structure of the biosphere is defined by its peculiar state of organization. Being an isolated part of the whole, the biosphere occupies a regularly fixed place within the planet'. [Ver97]

Indeed, influence of such geo- and helio-factors on different aspects of human activities turns out to be quite evident. At the same time, the opportunity of integrating the given natural-science facts and figures into a unified system directly influencing the bio-social activity of human beings appears only today - owing to the analytical potential of the newest technologies and approaches under consideration, which enable detection and full-scale consideration of the unobvious correlations in the information-specific flows.

Eventually, within the framework of economy-specific simulation the human being can be represented not from the standpoint of his isolation or even opposition to the natural processes of terrestrial and space scales, but rather in terms of his biosocial essence being thus characterized as an element of reality subject to quite evident social-and-economic impact as well as influenced by global processes of space level, reducible to specific indicators, which - based on Big Data techniques - can be 
successfully interpreted and used as individual cases while taking managerial decisions owing to correlation of the space-specific (biological) elements and social (economic) elements in the human conduct.

This bio-social human activity in the information interfacing system is manifested in various spheres that can be thoroughly studied with the aid of Big Data techniques - from the standpoint of possible impacting them with the aim of optimizing the process of managerial decision making and achieving the biggest possible economic effect.

In this context, we consider it quite reasonable to undertake intellectual analysis of a number of significant human bio-social activity spheres with the aid of Big Data techniques. These spheres could include environmental-geographical realm; approaching this realm would enable interpretation of the human social-economic activity as a derivative of the processes taking place in the geo- and helio-systems.

Analysis of spirituality and world outlook as manifestations of the people's activities will facilitate defining the influence of spiritual, religious and other forms of irrational perceptions on the social-and-economic sides of the people's life activities; it will also enable influence on the world outlook guidelines including (as individual case) new scientific achievements as related to collective consciousness.

In the sphere of educational preferences, the biggest interest is in the changes of educational priorities - with the view of determining policy in this sphere. The present-day demographic situation makes it necessary to turn to the family and marriage in order to detect tendencies of changes in perceiving the matrimonial relations and family values as well as views on different marriage forms.

Given all the complexity and manifoldness of financial process in the contemporary economy, the researchers are focused on the investment sphere, which implies addressing motivations and directions of the population's investment activity (forecasting possible alternatives of investing the available capital, revealing dependence of the lead-time on investment upon economic, social and natural trends).

Determining the marketing strategy - based on the Big Data approaches actualizes significance of the consumption sphere from the point of view of producers with regard to manufacturing different product groups and taking possible corrective measures. Of no less importance is significance of politics - from the point of view of analyzing passionate manifestations of communities' activities and associated possibility of constructive impact on the public consciousness with the purpose of optimizing management and preventing conflicts and eliminating social tensions.

Deviant activities and social concerns cannon be always clearly institutionalized. Despite this fact, analysis with the aid of Big Data can be quite successfully applied to this sphere, in this case, it will be possible to reveal the causes and conditions determining realization of individual's deviant inclinations, which would facilitate prevention of similar actions and ensure development of technologies of eliminating social concerns at the personal and public levels.

Against the described background, Big Data techniques perform as a kind of tool of regular recreation of the interactive reality and manipulation in the virtual environment, thus making it possible to transform in a very profound manner the perception of the information-related aspect of manifoldness of societal relations 
determining (in more precise terms - computing with the aid of large-scale data volume) the leitmotiv that directs specific phenomenon's or process's development vector. 'Webbed' in such a manner informational or digital reality, would enable appropriate decisions, thus submerging the person into the world of models and essential schemes, in which alteration of a given value can lead to a chain reaction and change the character of the whole 'reality' based on Big Data. Under these conditions, each indicator's value in the reality's 'digital vision' structure becomes more significant, otherwise interpretation of the situation will turn wrong and the respective decision - erroneous. Against this background, it is important to succeed in selecting from the variety of the Big Data-offered facts and figures those values that directly or indirectly affect the situation, simultaneously differentiating between data of primary and secondary importance, removing unnecessary information. This being so, it is appropriate to put the question of false knowledge occurrence, in which event the antecedent knowledge could be unnecessarily doubled and even cancelled; this would lead to impossibility of collecting qualitatively new knowledge as the next step in decision making and consequently - to failure of developing the Big Data-based reality.

It is possible to state formation of a new guideline, the principles of which significantly reduce topicality of the conventional question 'Why?' (which has been shaping the baseline of philosophical and scientific research for a long period) thus substituting this question for the questions 'How?' and 'What?', which are much more significant and meet the interests of the present-day society. At first sight, it might look as escaping causality, consequently - loss of scientific approach, rationality and feasibility.

In our opinion though, attentive consideration of the issue makes it possible to emphasize that deviation from searching for causality makes up merely outward appearances, because in reality the search for causality transforms and gives way to the use of huge data field, which combined with knowledgeable and correct problem setting and use of IT assures the XXI-century science transition to a qualitatively new level and in the practical sphere - enables weighed decisions and efficient economic modeling. In this connection, quantitative indicators will no longer act as transcendental quantities, they become immanent indicators of social-and-economic reality, being converted effectively into the cognition tool, into the tool of influence and perception, thus absolutizing the cause-and-effect relation, on the one hand, but remove them from the field of vision and make them unobvious, on the other hand.

As a result of using the Big Data techniques in the contemporary social-andeconomic conditions, opportunity is generated as to assigning a new status to the information - as a virtually inexhaustible economic resource, the significance of which leaves no doubts in society, while its infiniteness and universal nature are ensured by the possibility of practically endless number of interpretations. In these circumstances, it is extremely important to mention that - as distinct from other economic resources (the volume and quality of which facilitate taking this or that decision) - the information is not 'depreciated', it is never unfit for use, but owing to the abovementioned capacities and in the course of interpretational possibilities with the aid of Big Data can be incorporated into new information flows. Alongside with 
that, the information volume grows from year to year evidently demonstrating its inexhaustibility.

All that provides an opportunity of stating the fact that information is in the process of being converted into a multidimensional tool (of analysis, management and forecasting) affecting not only the up-to-the-minute interests and needs of the people, but requiring a deep philosophical comprehension - due to its orientation towards principal transformation of in-depth fundamentals of both gnoseological and (unconditionally) ontological planes, in any possible manifestations. Such processes require all-round analysis and development of distinctive and absolutely new theoretical platform, which in short-term perspective means raising the philosophy to a qualitatively new level linked to transformation of the whole problem-bearing research field, consequently providing for elaboration of new ontological principles and new methodology of comprehending the human's knowledge and existence.

\section{References}

1. Berezin SB. Cloud technologies in the natural sciences and humanities / S.B. Berezin, Big Data in the National Economy. Moscow, 2014. URL: http://istina.msu.ru/conferences/ presentations/8469122/

2. Mayer-Schonberger V, Cukier K. Big Data: A Revolution That Will Transform How We Live, Work and Think / Viktor Mayer-Schonberger, Kenneth Cukier. Trans. from English by Inna Gaydyuk. M.: Mann, Ivanov and Ferber, 2014; 232 p.

3. Sadovnichy VA. Intelligent System of Scientific-and-Technical Information Thematic Study (ISTINA) / S.A. Afonin and colleagues. Under the editorship of Academician V.A. Sadovnichy. M.: Moscow University Publishers, 2014; 262 p.

4. Tsiolkovsky KE. The Will of the Universe. The Unknown Intelligence. M.: AVT, 1928; $23 \mathrm{p}$.

5. Vernadsky VI. Scientific Thought as a Planetary Phenomenon./ Trans. from Russian by B.A.Starostin. M.: Nongovernmental Ecological V.I.Vernadsky Foundation, 1997; 265 p. 